\title{
Gut microbiota and the pathophysiology of cardiovascular disease
}

\section{Type}

Review paper (After Acceptance of EiC)

\section{Keywords}

cardiovascular diseases, heart failure, dysbiosis, gut microbiota, pathophysiology

\begin{abstract}
In recent years, significant findings with respect to the association of the gut microbiota and various human diseases have been discovered. The diversity can be explained by a multitude of interactions between intrinsic and environmental factors that are unique to each individual. This uniqueness of the microbiota may explain why some individuals are more prone to develop cardiovascular diseases. Gut dysbiosis plays a significant role in various pathophysiological processes. It can be postulated that health is linked to the homeostasis of the gastrointestinal microbiota. We provide an overview of diagnostic procedures to determine the microbiota's composition, the mechanisms of microbiota interactions and some effects of the microbiota on the development of cardiovascular diseases.
\end{abstract}




\section{Gut microbiota and the pathophysiology of cardiovascular disease}

2

3 Abstract

4 In recent years, significant findings with respect to the association of the gut microbiota and

5 various human diseases have been discovered. The diversity can be explained by a multitude of

6 interactions between intrinsic and environmental factors that are unique to each individual. This uniqueness of the microbiota may explain why some individuals are more prone to develop

8 cardiovascular diseases. Gut dysbiosis plays a significant role in various pathophysiological

9 processes. It can be postulated that health is linked to the homeostasis of the gastrointestinal microbiota. We provide an overview of diagnostic procedures to determine the microbiota's composition, the mechanisms of microbiota interactions and some effects of the microbiota on the development of cardiovascular diseases.

14 Key words: gut microbiota, dysbiosis, cardiovascular diseases, heart failure, pathophysiology. 
Abbreviations

\begin{tabular}{|c|c|}
\hline ABBREVIATION & DEFINITION \\
\hline GIT & gastrointestinal tract \\
\hline BMI & body mass index \\
\hline HDL & high-density lipoprotein \\
\hline CVD & cardiovascular diseases \\
\hline RNA & ribonucleic acid \\
\hline qPCR & quantitative polymerase chain reaction \\
\hline T-RFLP & terminal restriction fragment length polymorphism \\
\hline ARISA & automated method of ribosomal intergenic spacer analysis \\
\hline FISH & fluorescence in situ hybridization \\
\hline PAMPs & pathogen associated molecular patterns \\
\hline LPS & lipopolysaccharide \\
\hline TLRs & toll-like receptors \\
\hline PG & peptidoglycan \\
\hline SCFAs & short-chain fatty acids \\
\hline TMA & trimethylamine \\
\hline TMAO & trimethylamine $\mathrm{N}$-oxide \\
\hline hs-CRP & high-sensitivity C-reactive protein \\
\hline GPCRs & G-protein-coupled receptors \\
\hline LDL & low-density lipoprotein \\
\hline CKD & chronic kidney disease \\
\hline BSH & bacterial bile-salt hydrolase \\
\hline oxLDL & oxidized low-density lipoprotein \\
\hline GFD & gluten free diet \\
\hline
\end{tabular}

17 


\section{INTRODUCTION}

21 In the last ten years, significant advancements have been made in understanding the human microbiota and its role in various diseases (1). Gut microbiota is the collection of bacteria, fungi, viruses, archaea, and parasites in the gastrointestinal tract (GIT), producing a diverse ecosystem of about $10^{14}$ microorganisms. A direct connection to our microbiota composition are our eating habits. As an illustration, epidemiological evidence on immigrants suggests that there is a potential fourfold increase in obesity risk within fifteen years of emigrating to the U.S. compared to populations remaining in their birth country. This can be furthermore accompanied by a decrease in their gut microbial diversity and function $(2,3)$. It has been shown that environmental factors (e.g., diet, household cohabitation) greatly outweigh heritable genetic contributions to the composition and function of gut microbiota (4). Furthermore, Rothschild et al. constructed a microbiomeassociation index that mimics heritability statistics $(4,5)$. The most significant described associations were between the gut microbiome and host phenotypes for body mass index (BMI), waist-to-hip ratio, fasting glucose levels, glycemic status, high-density lipoprotein (HDL) cholesterol levels, and monthly lactose consumption $(4,5)$. If the homeostasis of the gut microbiota, which acts almost like an endocrine organ, is disturbed, dysbiosis can contribute to the development of various diseases $(6,7)$.

It comes as no surprise that some of these potential diseases include cardiovascular diseases (CVD), chronic kidney disease, type 2 diabetes mellitus, non-alcoholic fatty liver disease, and even certain types of cancer $(1,8-10)$. The term dysbiosis presents a change in the composition of the gut microbiota. Reasons for such a change are manifold and can range from exposure to several factors (diet, increased stress, antibiotic usage). Ilya Ilyich Mechnikov (also written as Élie Metchnikoff) coined the term dysbiosis at the beginning of the $20^{\text {th }}$ century. Together with Paul Ehrlich, they were awarded in 1908 the Nobel Prize in Physiology or Medicine "in recognition of their work on immunity". Dysbiosis might offer an explanation as to why certain individuals are more susceptible 
to develop specific diseases. Moreover, it has recently been recognized that dysbiosis increases the chances for developing atherosclerosis and hypertension $(1,9,11)$.

Two authors (P.S. and K.S.) performed an electronic bibliographic search of the PUBMED and Cochrane databases. The databases were primarily searched using the keywords/MeSH terms "cardiovascular diseases", "gastrointestinal microbiome" and "dysbiosis" with various subheadings, taking into account the latest findings (last five years) with exceptions when citing older original findings. The initial search resulted in 231 entries were further screened by applying additional filters and eligibility criteria (full text, books and documents, clinical trial, meta-analysis, randomized controlled trial, systematic review, in the last five years). This alongside the exclusion criteria (exclusion of studies, chapters and articles with similar findings published as different bibliographic units) narrowed the final result to 28 articles. During analysis of these articles the therein reported and thematically relevant articles were also included. The inquiry was performed without time restriction at the Research department of the University clinical center Maribor.

\section{DIAGNOSTIC PROCEDURES FOR DETERMINING THE COMPOSITION OF THE GASTROINTESTINAL MICROBIOTA}

The composition of the microbiota, its diversity and potential significance in maintaining homeostasis of epithelial cell function, prevention of pathogenic microorganism growth and production of different substances as well as ingredients can be determined with the use of a variety of methods, which differ in resolution (11-13). These methods may be employed to compare and specify the microbiota composition between samples, determine the specific microorganisms, their intercellular relationships and dependencies as well as their role in metabolism on basis of their genetic information (14). Some approaches and the corresponding terms can be seen in table 1 $(15,16)$. 
An accurate representation of the human microbiota composition, as well as its characterization, was one of the main goals of the quite recent "Metagenomics of the Human Intestinal tract project" $(17,18)$. Methods for defining the microbiota composition can be divided into traditional and molecular. Commonly known traditional methods are "the counting of cells on a specific culture medium" and the "most probable number" (14). Culturing methods have certain important drawbacks and restrictions, namely: a large amount of laboratory work, limited culturing possibility, and range (only $30 \%$ of the intestinal microbiota) (14). It has to be stressed that successful growth can be observed during cultivation only in $0.01-10 \%$ of the cells in the microbiological sample. Most molecular techniques utilize the ribosomal 16S and 18S RNA (rRNA), which function as phylogenetic markers for the taxonomic classification of organisms and are preserved in all bacteria, archaebacteria, and eukaryotes. Some molecular methods include:

- quantitative polymerase chain reaction (qPCR) - amplification and quantification of 16S rRNA, which enables the phylogenetic identification of microbiota.

- denaturing gradient gel electrophoresis - analysis of microbial communities by the sequence-specific separation of PCR-amplified 16S rRNA fragments by using a linear gradient of denaturants or temperature.

- terminal restriction fragment length polymorphism (T-RFLP) - the amplification is performed with one or both the primers having their 5' end labeled with a fluorescent molecule with a subsequent restriction of 16S rRNA products with enzymes and gel electrophoresis separation

- automated method of ribosomal intergenic spacer analysis (ARISA) - PCR multiplication of a region between the $16 \mathrm{~S}$ and $23 \mathrm{~S}$ RNA regions, with subsequent fragment separation via capillary electrophoresis.

- Fluorescence in situ hybridization (FISH) - hybridization of oligonucleotides marked with a fluorescent molecule with $16 \mathrm{~S}$ genes with subsequent measuring of fluorescence via a flow cytometer. 
- DNA-microarray - hybridization of oligonucleotide probes, marked with a fluorescent molecule, with complementary oligonucleotides and subsequent measuring of fluorescence with a laser.

- $\quad$ sequencing of cloned 16S rRNA genes - cloning of all the 16S rRNA products, Sanger sequencing and capillary electrophoresis.

- sequencing of 16S rRNA products - also known as deep sequencing of 16S rRNA products.

- shotgun metagenomics sequencing of the whole microbiome.

- shotgun metatranscriptomics sequencing for determining gene expression of the microbiota.

All of the mentioned methods have their advantages as well as drawback, which become evident either when determining phylogenetic differences or in form of accurate identification, accessibility, and of course price.

\section{MECHANISMS OF MICROBIOTA ACTIVITY}

One of the major risk factors for CVD is atherosclerosis. Its pathophysiological basis is the accumulation of cholesterol, followed by an immune response that leads to the formation of plaques $(1,11,19)$. Gut dysbiosis can via modulation of the inflammatory response as well as production of microbial metabolites accelerate this process (20-22):

a) gut dysbiosis and atherosclerosis: The GIT acts as a barrier, any changes in its permeability can lead to complications. These changes are associated with the reduced expression of tight junction proteins (e.g., zonula occludens-1, claudin-1 and occluding) and an imbalance between epithelial cell death and regeneration $(1,21,22)$. What follows is the translocation of bacteria, which stimulate, via the recognition of their pathogen associated molecular patterns (PAMPs), an immune response and inflammation. What is 
more, lipopolysaccharide (LPS) and peptidoglycan, which are part of the cell wall, have also been described as risk contributing factors.

I. Humans: The correlation between LPS and CVD risk was first proposed in 1999. This has been done via measurements of plasma endotoxin levels (21). The correlation of endotoxemia and CVD burden has been confirmed in some studies. Cani et al. reported the correlation between dysbiosis and the suppressed expression of tight junction proteins, which in turn leads to the above described cascade and translocation of LPS into the blood (23). On the pathophysiological level, it has been proposed that the (gut dysbiosis-derived) LPS might act as a modulator of toll-like receptors (TLRs), which are mostly present on immune sentinel cells who are responsible for the immune systems defense mechanisms. The upregulation of these proteins has been associated with an inflammatory activation which in turn promoted the process of atherosclerosis. The bacterial cell wall component peptidoglycan (PG) can apparently also impair the intestinal epithelial barrier via an inflammatory response. This has been demonstrated in patients with over-representation of genes for PG synthesis. Furthermore, this polymer might be responsible for more vulnerable plaques in sclerotic arteries $(11,19)$. Inflammatory processes can be also stimulated by other PAMPs (CpG oligodeoxynucleotides flagellin, lipopeptides etc.). All in all, the scientific results in the last years have confirmed the correlation of the gut microbiota and atherosclerosis risk $(1,11,24)$,

b) gut microbial metabolites in atherosclerosis: in the metabolism of intestinal bacteria, different metabolites are produced that show involvement in the pathophysiology of atherosclerosis (Figure 1).

Short-chain fatty acids (SCFAs) play a significant role in the development of metabolic diseases. Bacteria can via the use of choline-specific and carnitine-specific trimethylamine (TMA) lyases 
form TMA which in turn gets after absorption transferred to the liver. Thought further metabolic processes (flavin-containing monooxygenases) the TMA gets converted into trimethylamine Noxide (TMAO) $(11,22)$. TMAO has according to literature a variety of different mechanism which all promote atherosclerosis (cholesterol influx, cholesterol efflux inhibition, bile acids (BA) pathway blockade, excessive activation of platelets) $(1,11)$. According to researchers, TMAO could represent, in addition to the role of a biomarker for CVD and atherosclerosis, a potential therapeutic target in the future.

c) gut microbiota and hypertension: already in 1982, Honour et al. showed that blood pressure could be elevated by the use of antibiotic treatment (20).

I. Animals: A study of Yang et al. from 2015 in spontaneously hypertensive rats confirmed that altering gut microbiota (e.g., decreasing/increasing) can influence the regulation of blood pressure. Specifically stressed was the increase in the ratio of Firmicutes/Bacteroidetes species (11).

II. Humans: Current evidence, even though it might not yet be complete, has elucidated and shown the importance of SCFAs and oxidized low-density lipoprotein (ox-LDL) in hypertension. The microbiota of a person is very specific and stable throughout the adult life span, despite the fact that $90 \%$ of them are dominated by representatives of only two bacterial species, Firmicutes and Bacteroides. Bacteria of these two species are good structural polysaccharides and SCFAs producers (e.g., butyrate, acetate, proprionate). They are crucial for the homeostasis of the gut microbiome and host immunity $(1,11,13)$. Interesting is the fact that different bacteria form different types of SCFAs. The study from GomezArango et al. has shown that in obese pregnant women an increase in butyrateproducing bacteria (Lachnospiraceae, Ruminococcaceae, and Acidaminococcaceae families) is associated with lower blood pressure (22). SCFAs can stimulate host G-protein-coupled receptors (GPCRs)-regulated pathways to affect renin secretion and therefore blood pressure. From a 
physiological basis the blood pressure regulatory mechanisms are primarily dependent on vasoconstriction and vasodilation. Another fascinating mechanism is low-density lipoprotein (LDL) oxidation by bacteria which causes excessive vasoconstriction. This is also promoted by pro-inflammatory cytokine expression, that causes oxidative stress and stimulates this process (1). All in all, higher levels of oxLDL can lead to a vasodilator/vasoconstrictor production disequilibrium,

d) gut microbiota and heart failure: with a growing body of scientific evidence the link between the gut in the pathogenesis of heart failure, the so-called "gut hypothesis of heart failure" is becoming more and more plausible $(23,25,26)$. The pathophysiological events are shown in figure 2 .

I. Humans: In a fascinating study from Niebauer et al. it has been shown that heart failure patients who had an accompanying peripheral edema exhibited increased concentrations of plasma inflammatory markers (endotoxin, cytokines) in comparison with those without an edema $(11,23)$. When patients received diuretic treatment (short-term), serum concentrations of endotoxin, but not cytokines decreased. Furthermore, in a different study, higher serum concentrations of immunoglobulin A - anti-lipopolysaccharide were seen in individuals with heart failure and a lower intestinal blood flow. Surprisingly the microbiota was different in these individuals in comparison with the control group (11). Moreover, studies have also shown that TMAO levels were elevated in patients with heart failure in comparison with the control group. TMAO levels exhibited a remarkably strong adverse prognostic value in a cohort of stable patients with heart failure.

e) gut microbiota and myocardial infarction: Studies showed that atherosclerotic plaques (especially vulnerable/instable ones) can contain bacterial DNA.

I. Humans: In those specific individuals the bacterial species found in the plaques was then also found in the GIT $(11,25,26)$. Subsequently, this means that the 
composition of ones' microbiota might be a reason for increased rate as well as instability of plaque formation.

II. Animals: Lam et al. studied the impact of gut microbiota composition and the severity of myocardial infarction in rats $(11,27)$. The authors reported that the levels of leptin and other catabolic amino acid metabolites as well as the myocardial infarct size were lower when rats were given broad-spectrum antibiotics (27). What is more, administration of Lactobacillus plantarum showed after a myocardial infarction in rodents. A different study showed that the addition of Lactobacillus rhamnosus GR-1 attenuated left ventricular hypertrophy and heart failure after experimental myocardial infarction (28).

gut microbiota and chronic kidney disease: CVD and kidney diseases are closely interrelated (e.g., cardiorenal syndrome). Patients with chronic kidney disease (CKD) have a greater risk of CVD complications as well as an increased mortality rate, therefore, in many research environments, they are trying to identify the most appropriate biomarkers of potential complications (29). Studies have confirmed that patients with CKD have a distinctly different composition of gut microbiota. In CKD an influx of circulating urea and other uremic toxins into the gut lumen occurs and induces the so-called "leaky gut" $(11,30-34)$. The pathophysiological mechanism is shown in figure 3.

I. Humans: Recently, the DNA of gut microbiota has been detected in the plasma of CKD patients on chronic hemodialysis using bacterial 16S rDNA amplification and DNA pyrosequencing. Moreover, the levels of the bacterial DNA correlated with increased plasma inflammatory marker levels. Poorly dialyzable proteinbound uremic toxins such as indoxyl sulfate and p-cresyl sulfate are associated with poor cardiovascular outcomes $(11,32)$. TMAO has been known to accumulate in the plasma of patients with CKD, and higher TMAO levels were associated with 
Research has also been focused on links between dysbiosis and obesity, type 2 diabetes, and dyslipidemia. Obesity has been linked to a higher ratio of Firmicutes to Bacteroidetes, type 2 diabetes was associated with a reduction in butyrate-producing bacteria and an increase of Lactobacillus spp $(1,10,11,19,24)$. Gut microbiota have via their own enzymes (e.g., bacterial bilesalt hydrolase $(\mathrm{BSH})$ ) the ability to regulate BA metabolism. This is essential for the formation of secondary BAs. The decrease of mentioned BSH activity in a dysbiotic ecosystem leads to a variety of pro-atherosclerotic effects. Specifically, dysbiosis can lead to impaired cholesterol elimination and dyslipidemia by modulating hepatic and/or systemic lipid metabolism, as well as glucose metabolism $(1,11,19,24)$.

\section{CLINICAL RELEVANCE OF GUT MICROBIOTA}

245 Over the last decade, knowledge about the relationship between dysbiosis and the pathogenesis of

246 CVD has rapidly accumulated (36-39). Some of the lessons are new opportunities for early,

247 targeted action, and at the same time, many research questions are being raised about the relationship between "what is cause and what is the consequence" and therapeutic options. Research on dysbiosis in some groups of patients is surprising, typical signs of disrupted microbiota are reduced diversity, a decrease in anti-inflammatory species such as Faecalibacterium prausnitzii and an increase in various members of the Enterobacteriaceae $(40,41)$. Mahnic et al. have confirmed that bacterial and fungal alterations of the gut microbiota, which are often reported to be disease-specific, such as a decrease of Faecalibacterium and an increase in E. coli, Enterococci and Candida, are often found in a broader population of hospitalized patients with different diseases and also in healthy controls (41). Furthermore, the authors showed a prominent correlation between levels of C-reactive protein and the abundance of Enterococcus. Although gut dysbiosis is often perceived as random, the research group has described two different types in 
which the severity of the disorder was correlated with specific microbial patterns, the degree of inflammation and, to some extent, the use of antibiotics (41). Specifically, the clinical examples of gut microbiota interventions for CVD can be divided into multiple groups: 1) dietary interventions; 2) exercise; 3) pro-, pre-, antibiotics; 4) fecal transplantation; 5) TMAO reduction 6) other (e.g., nanomedical approaches) $(7,42)$.

Modulating the gut microbiota with the help of dietary changes has been shown to be a promising intervention for lowering the risk for coronary diseases $(43,44)$ as well as general atherosclerosis (19). A Mediterranean diet intervention have been reported to alter the gut microbiome in older people and thus reduce frailty and improving health status (45). Furthermore, in animal models high fiber diet has been associated with lower blood pressure, lower cardiac hypertrophy and lower degree of fibrosis (46). Even trace elements as zinc have shown to have a significant impact on the homeostasis of the microbiota (47). The habitual diet of a person is considered a key driver in establishing this core microbial profile (48). Acute dietary interventions in humans lead to transient microbial shifts (e.g., days to weeks) (49). Moreover, for quite some time gluten free diet (GFD) plans have been trending in the general unaffected populous as a healthy diet change, despite being primarily aimed at those with gluten-related disorders (e.g., celiac disease, gluten allergy, etc.) (50). Many studies tried to evaluate the impact of such a dietary change (51-54). Some of the commonly reported changes include a reduction in E. hallii, A. hadrus, Bifidobacterium and an increase in Enterobacteriaceae and Escherichia coli (55). It has been described that the effects of GFD, while reducing bacterial richness, heavily depend on the subject's health as well as disease state (e.g., celiac disease, healthy) (55). Reports on this matter differ based on study population, geographical diversity as well as the individuality of patients. Recent studies report that in normal subjects the diet had deleterious effects $(54,55)$ and that the opposite was the case in patients with celiac disease $(55,56)$. Furthermore, as stated by Lebwohl et at, avoidance of gluten in healthy subjects may result in reduced consumption of beneficial whole grains, which may affect cardiovascular risk (57). It has to be stressed that the results from the effect of GFD on health and the gut microbiota cannot be 
extrapolated from one population (or region) to others nor are they universally applicable (58). This statement applies to all dietary intervention in any other dysbiosis associated gastrointestinal disease (e.g., inflammatory bowel disease). Such alterations should not be done lightly.

Nevertheless, it is generally considered that irregular eating habits, such as skipping breakfast, having dinner late, and late-night eating, contribute to obesity and other metabolic disorders (59).

Exercise is of the outmost importance for a heathy human being. Not only does it lower the risk for CVD and improves long term survival in patients with preexisting heart conditions (60), it has also been shown that regular exercise promotes a healthy gut microbiota while protecting the permeability and function of the gut barrier (61). Several studies indicate that exercise leads to an increase in the number of health-promoting bacterial species (62-64). For example, in active women a higher abundance of (Faecalibacterium prausnitzii, Roseburia hominis and Akkermansia muciniphila) has been shown (64). However, based on the Allen, et al. (65) the effects of exercise on the gut microbiota depend on the continuity of exercise and are therefore reversible.

Other modalities of microbiota modulation include probiotics (66), prebiotics (67), postbiotics as well as antibiotics (68). Probiotics are live microorganisms administered to re-establish an intestinal ecological balance, through a variety of different mechanisms (68), which also include immunomodulation of the host and inhibition of bacterial toxin production. Therapy with probiotics has shown promise in patients with impaired cardiac function $(68,69)$ and have been associated with a protective effect against colorectal cancer (67). The by-products of probiotic cultures are called postbiotics. These, despite only recently getting attention, have been shown exhibit positive effects (e.g., suppress colonic inflammation and restore gut barrier integrity) (67). However, the exact identity of the postbiotics and the molecular mechanisms are not yet fully understood (67). Moreover, prebiotics have been reported to beneficially modify lipid metabolism (70). The use of antibiotics to specifically alter the microbiota is, due to a wide range of potential side-effects, still debatable. Antibiotic administration presents the most aggressive means to 
manipulate gut microbiota composition. Negative effects include the depletion of bacterial

313 diversity, altered gene expression and metabolism, selection for intrinsically resistant bacteria etc.

314 (71,72). That is why antibiotics have also been referred to as deep modulators of the gut microbiota

315 (figure 4) (72,73). Some examples of modulation include the study on obesity (74), insulin

316 resistance, diabetes (75), myocardial infarction (mentioned previously) (27). Mouse models

317 showed that the effects of antibiotic treatment towards weight appears to depend on several factors

318 (e.g., drug dosage, timing of exposure) (74). The results depending on dosage showed either a

319 tendency to become underweight (76) or overweight (75). This has been explained as selective

320 dysbiosis. At the same time, certain antibiotics showed in obese mice antidiabetic effects (77).

321 Another study provided evidence that early life treatment of mice with vancomycin was beneficial

322 in preventing the onset of diabetes by an increase in health-promoting bacteria (78). In human studies antibiotic exposure during infancy was linked to being a risk factor for becoming overweight later in childhood (79). These results still need to be validated by additional studies (80).

326

Fecal microbiota transplantation has already therapeutically confirmed the importance of a healthy gut microbiota in certain patients. This form of treatment is several decades old and still presents an important intervention (81). It has been even shown that this method might improve insulin resistance (82). Due to the negative effect of TMAO, probiotics as well as other pharmacological interventions in form of TMAO reduction inhibitors can be utilized to inhibit or block specific microbial metabolic pathways. In mice the treatment with a TMA-lyase inhibitor has shown promise by improving hemodynamical parameters (83). However, further studies will have to be performed to fully determine the safety profiles and possible consequences of such therapies.

Microbiota and dysbiosis represent areas of research interest that will most certainly change some 
339 of cardiovascular diseases. The present article has discussed different aspects of dysbiosis, its

340 pathophysiological pathways and its effects on cardiovascular health as well as possible promising

341 interventions. All of the presented methods alter the microbial composition in different ways (e.g.,

342 suppression of TMA, increase in beneficial cultures etc.) and may lead to positive changes that help

343 prevent and/or reduce deleterious effects of atherosclerosis, hypertension, hearth failure, obesity as

344 well as diabetes. The presented changes have in certain cases still only been reported in animal

345 models and should therefore not be directly extrapolated to humans. Furthermore, although we can

346 change the composition of the microbiota, unfortunately at the present moment we cannot fully

347 predict the long-term effects of our actions as well as offer universal guidelines for all

348 interventions. 
350 Author contributions: Both authors equally contributed to this paper with conception and design

351 of the study, literature review and analysis, drafting and critical revision and editing, and final

352 approval of the final version.

353 Declaration of conflicting interests: The authors declare that there is no conflict of interest.

354 Funding: The authors received no financial support for the research, authorship, and/or publication 355 of this article.

356

357 


\section{REFERENCES}

359 1. Lau K, Srivatsav V, Rizwan A et al. Bridging the Gap between Gut Microbial Dysbiosis 360 and Cardiovascular Diseases. Nutrients. 2017;9(8):859.

361 2. Vangay P, Johnson AJ, Ward TL et al. US Immigration Westernizes the Human Gut $362 \quad$ Microbiome. Cell. 2018;175(4):962-972.e10.

3. Lauderdale DS, Rathouz PJ. Body mass index in a US national sample of Asian Americans: Effects of nativity, years since immigration and socioeconomic status. Int J Obes. 2000;24(9):1188-94.

4. Hills RD, Pontefract BA, Mishcon HR et al. Gut microbiome: Profound implications for diet and disease. Nutrients. 2019;11(7):1613.

5. Rothschild D, Weissbrod O, Barkan E et al. Environment dominates over host genetics in shaping human gut microbiota. Nature. 2018;555(7695):210-5.

6. Szychlinska MA, Di Rosa M, Castorina A, Mobasheri A, Musumeci G. A correlation between intestinal microbiota dysbiosis and osteoarthritis [Internet]. Vol. 5, Heliyon. Elsevier; 2019. p. e01134.

7. $\mathrm{Xu} \mathrm{H}$, Wang X, Feng $\mathrm{W}$ et al. The gut microbiota and its interactions with cardiovascular disease. Microb Biotechnol. 2020;13(3):637-56.

8. Nallu A, Sharma S, Ramezani A, Muralidharan J, Raj D. Gut microbiome in chronic kidney disease: challenges and opportunities. Transl Res. 2016/04/30. 2017;179:24-37.

9. Tang WHW, Wang Z, Levison BS et al. Intestinal microbial metabolism of phosphatidylcholine and cardiovascular risk. N Engl J Med. 2013;368(17):1575-84.

10. Ismail NA, Ragab SH, ElBaky AA et al. Clinical research $<$ br $>$ Frequency of Firmicutes and Bacteroidetes in gut microbiota in obese and normal weight Egyptian children and adults. Arch Med Sci. 2011;7(3):501-7.

11. Tang WHW, Kitai T, Hazen SL. Gut Microbiota in Cardiovascular Health and Disease. Circ Res. 2017;120(7):1183-96.

12. Sekirov I, Russell SL, Antunes LCM, Finlay BB. Gut Microbiota in Health and Disease. 
Physiol Rev. 2010;90(3):859-904.

13. Tamburini S, Shen N, Wu HC, Clemente JC. The microbiome in early life: implications for health outcomes. Nat Med. 2016;22:713.

14. Š Šket R, Prevoršek Z, Košeto D et al. Analitski in konceptualni izzvi pri raziskovanju

15. Marchesi JR, Ravel J. The vocabulary of microbiome research: a proposal. Microbiome. 2015;3(1):31.

16. Durack J, Lynch S V. The gut microbiome: Relationships with disease and opportunities for therapy. J Exp Med. 2018/10/15. 2019;216(1):20-40.

17. Turnbaugh PJ, Ley RE, Hamady M et al. The human microbiome project. Nature. 2007;449(7164):804-10.

18. Qin J, Li R, Raes J et al. A human gut microbial gene catalogue established by metagenomic sequencing. Nature. 2010;464(7285):59-65.

19. Yamashiro K, Tanaka R, Urabe T et al. Gut dysbiosis is associated with metabolism and systemic inflammation in patients with ischemic stroke. PLoS One. 2017;12(2):e0171521.

20. Qi Y, Aranda JM, Rodriguez V, Raizada MK, Pepine CJ. Impact of antibiotics on arterial blood pressure in a patient with resistant hypertension - A case report. Int J Cardiol. 2015/07/30. 2015;201:157-8.

21. Niebauer J, Volk H-D, Kemp M et al. Endotoxin and immune activation in chronic heart failure: a prospective cohort study. Lancet. 1999;353(9167):1838-42.

22. Gomez-Arango LF, Barrett HL, McIntyre HD et al. Increased Systolic and Diastolic Blood Pressure is Associated with Altered Gut Microbiota Composition and Butyrate Production in Early Pregnancy. Hypertension. 2016;68(4):974-81.

23. Cani PD, Amar J, Iglesias MA et al. Metabolic Endotoxemia Initiates Obesity and Insulin Resistance. Diabetes. 2007;56(7):1761 LP - 1772.

24. Brown JM, Hazen SL. The gut microbial endocrine organ: bacterially derived signals 
driving cardiometabolic diseases. Annu Rev Med. 2015;66:343-59.

25. Koren O, Spor A, Felin J et al. Human oral, gut, and plaque microbiota in patients with atherosclerosis. Proc Natl Acad Sci U S A. 2010/10/11. 2011;108 Suppl(Suppl 1):4592-8.

26. Ott SJ, El Mokhtari NE, Musfeldt M et al. Detection of diverse bacterial signatures in atherosclerotic lesions of patients with coronary heart disease. Circulation.

27. Lam V, Su J, Hsu A et al. Intestinal Microbial Metabolites Are Linked to Severity of Myocardial Infarction in Rats. PLoS One. 2016;11(8):e0160840.

28. Gan XT, Ettinger G, Huang CX et al. Probiotic administration attenuates myocardial hypertrophy and heart failure after myocardial infarction in the rat. Circ Hear Fail. 2014;7(3):491-9.

29. Abdelsalam L, Ibrahim AA, Shalaby A et al. Expression of miRNAs-122, -192 and -499 in end stage renal disease associated with acute myocardial infarction. Arch Med Sci. 2019;15(5):1247-53.

30. Gansevoort RT, Correa-Rotter R, Hemmelgarn BR et al. Chronic kidney disease and cardiovascular risk: epidemiology, mechanisms, and prevention. Lancet. 2013;382(9889):339-52.

31. Shi K, Wang F, Jiang H et al. Gut Bacterial Translocation May Aggravate Microinflammation in Hemodialysis Patients. Dig Dis Sci. 2014;59(9):2109-17.

32. Lin C-J, Chen H-H, Pan C-F et al. p-cresylsulfate and indoxyl sulfate level at different stages of chronic kidney disease. J Clin Lab Anal. 2011;25(3):191-7.

33. Tang WHW, Wang Z, Kennedy DJ et al. Gut microbiota-dependent trimethylamine N-oxide (TMAO) pathway contributes to both development of renal insufficiency and mortality risk in chronic kidney disease. Circ Res. 2014/11/05. 2015;116(3):448-55.

34. Tang WHW, Wang Z, Fan Y et al. Prognostic value of elevated levels of intestinal microbegenerated metabolite trimethylamine-N-oxide in patients with heart failure: refining the gut hypothesis. J Am Coll Cardiol. 2014/10/27. 2014;64(18):1908-14. 
35. Rhee EP, Clish CB, Ghorbani A et al. A combined epidemiologic and metabolomic approach improves CKD prediction. J Am Soc Nephrol. 2013/05/16. 2013;24(8):1330-8.

36. Zhu Q, Gao R, Zhang Y et al. Dysbiosis signatures of gut microbiota in coronary artery disease. Physiol Genomics. 2018;50(10):893-903.

37. Zhernakova A, Kurilshikov A, Bonder MJ et al. Population-based metagenomics analysis reveals markers for gut microbiome composition and diversity. Science (80- ). 2016/04/28. 2016;352(6285):565-9.

38. Belizário JE, Faintuch J. Microbiome and Gut Dysbiosis. In: Silvestre R, Torrado E, editors. Experientia supplementum (2012). Cham: Springer International Publishing; 2018. p. 45976.

39. Kriss M, Hazleton KZ, Nusbacher NM, Martin CG, Lozupone CA. Low diversity gut microbiota dysbiosis: drivers, functional implications and recovery. Curr Opin Microbiol. 2018/07/20. 2018;44:34-40.

40. Mahnic A, Rupnik M. Different host factors are associated with patterns in bacterial and fungal gut microbiota in Slovenian healthy cohort. PLoS One. 2018;13(12):e0209209e0209209.

41. Mahnic A, Breskvar M, Dzeroski S et al. Distinct Types of Gut Microbiota Dysbiosis in Hospitalized Gastroenterological Patients Are Disease Non-related and Characterized With the Predominance of Either Enterobacteriaceae or Enterococcus [Internet]. Vol. 11, Frontiers in Microbiology. 2020. p. 120.

42. Kazemian N, Mahmoudi M, Halperin F, Wu JC, Pakpour S. Gut microbiota and cardiovascular disease: opportunities and challenges. Microbiome. 2020;8(1):36.

43. Threapleton DE, Greenwood DC, Evans CEL et al. Dietary fibre intake and risk of cardiovascular disease: systematic review and meta-analysis. BMJ. 2013;347:f6879-f6879.

44. Aljuraiban GS, Griep LMO, Chan Q et al. Total, insoluble and soluble dietary fibre intake in relation to blood pressure: the INTERMAP Study. Br J Nutr. 2015/09/02. 2015;114(9):1480-6. 
45. Ghosh TS, Rampelli S, Jeffery IB et al. Mediterranean diet intervention alters the gut microbiome in older people reducing frailty and improving health status: the NU-AGE 1year dietary intervention across five European countries. Gut. 2020;69(7):1218 LP - 1228.

46. Marques FZ, Nelson E, Chu PY et al. High-fiber diet and acetate supplementation change the gut microbiota and prevent the development of hypertension and heart failure in hypertensive mice. Circulation. 2017;135(10):964-77.

47. Cerasi M, Ammendola S, Battistoni A. Competition for zinc binding in the host-pathogen interaction. Front Cell Infect Microbiol. 2013;3:108.

48. Leeming ER, Johnson AJ, Spector TD, Le Roy CI. Effect of Diet on the Gut Microbiota: Rethinking Intervention Duration. Nutrients. 2019;11(12):2862.

49. Tebani A, Bekri S. Paving the Way to Precision Nutrition Through Metabolomics [Internet]. Vol. 6, Frontiers in Nutrition . 2019. p. 41.

50. Ontiveros N, Rodríguez-Bellegarrigue CI, Galicia-Rodríguez G et al. Prevalence of SelfReported Gluten-Related Disorders and Adherence to a Gluten-Free Diet in Salvadoran Adult Population. Int J Environ Res Public Health. 2018;15(4):786.

51. Moszak M, Szulińska M, Bogdański P. You Are What You Eat-The Relationship between Diet, Microbiota, and Metabolic Disorders-A Review. Nutrients. 2020;12(4):1096.

52. Bonder MJ, Tigchelaar EF, Cai X et al. The influence of a short-term gluten-free diet on the human gut microbiome. Genome Med. 2016;8(1):45.

53. Garcia-Mazcorro JF, Noratto G, Remes-Troche JM. The Effect of Gluten-Free Diet on Health and the Gut Microbiota Cannot Be Extrapolated from One Population to Others. Nutrients. 2018;10(10):1421.

54. Hansen LBS, Roager HM, Søndertoft NB et al. A low-gluten diet induces changes in the intestinal microbiome of healthy Danish adults. Nat Commun. 2018;9(1):4630.

55. Caio G, Lungaro L, Segata N et al. Effect of Gluten-Free Diet on Gut Microbiota Composition in Patients with Celiac Disease and Non-Celiac Gluten/Wheat Sensitivity. Vol. 12, Nutrients . 2020. 
56. Nistal E, Caminero A, Vivas S et al. Differences in faecal bacteria populations and faecal bacteria metabolism in healthy adults and celiac disease patients. Biochimie. 2012;94(8):1724-9.

57. Lebwohl B, Cao Y, Zong G et al. Long term gluten consumption in adults without celiac disease and risk of coronary heart disease: prospective cohort study. BMJ. 2017;357:j1892j1892.

58. Pasolli E, Asnicar F, Manara S et al. Extensive Unexplored Human Microbiome Diversity Revealed by Over 150,000 Genomes from Metagenomes Spanning Age, Geography, and Lifestyle. Cell. 2019/01/17. 2019;176(3):649-662.e20.

59. Lopez-Minguez J, Gómez-Abellán P, Garaulet M. Timing of Breakfast, Lunch, and Dinner. Effects on Obesity and Metabolic Risk. Nutrients. 2019;11(11):2624.

60. Orimoloye OA, Kambhampati S, Hicks AJ et al. Higher cardiorespiratory fitness predicts long-term survival in patients with heart failure and preserved ejection fraction: The Henry Ford exercise Testing (FIT) project. Arch Med Sci. 2019;15(2):350-8.

61. Fiuza-Luces C, Santos-Lozano A, Joyner M et al. Exercise benefits in cardiovascular disease: beyond attenuation of traditional risk factors. Nat Rev Cardiol. 2018;15(12):73143.

62. Petriz BA, Castro AP, Almeida JA et al. Exercise induction of gut microbiota modifications in obese, non-obese and hypertensive rats. BMC Genomics. 2014;15(1):511.

63. Lambert JE, Myslicki JP, Bomhof MR et al. Exercise training modifies gut microbiota in normal and diabetic mice. Appl Physiol Nutr Metab. 2015;40(7):749-52.

64. Bressa C, Bailén-Andrino M, Pérez-Santiago J et al. Differences in gut microbiota profile between women with active lifestyle and sedentary women. PLoS One. 2017;12(2):e0171352.

65. Allen JM, Mailing LJ, Niemiro GM et al. Exercise Alters Gut Microbiota Composition and Function in Lean and Obese Humans. Med Sci Sports Exerc. 2018;50(4):747-57.

66. Nobili V, Putignani L, Mosca A et al. Bifidobacteria and lactobacilli in the gut microbiome 
of children with non-alcoholic fatty liver disease: which strains act as health players? Arch Med Sci. 2018;14(1):81-7.

522 67. Fong W, Li Q, Yu J. Gut microbiota modulation: a novel strategy for prevention and treatment of colorectal cancer. Oncogene. 2020;39(26):4925-43.

68. Markowiak P, Ślizewska K. Effects of probiotics, prebiotics, and synbiotics on human health. Nutrients. 2017;9(9):1021.

69. Costanza AC, Moscavitch SD, Faria Neto HCC, Mesquita ET. Probiotic therapy with Saccharomyces boulardii for heart failure patients: A randomized, double-blind, placebo-

70. Korcz E, Kerényi Z, Varga L. Dietary fibers, prebiotics, and exopolysaccharides produced by lactic acid bacteria: potential health benefits with special regard to cholesterol-lowering effects. Food Funct. 2018;9(6):3057-68.

71. Francino MP. Antibiotics and the human gut microbiome: Dysbioses and accumulation of resistances [Internet]. Vol. 6, Frontiers in Microbiology. 2016. p. 1543.

72. Bhalodi AA, van Engelen TSR, Virk HS, Wiersinga WJ. Impact of antimicrobial therapy on the gut microbiome. J Antimicrob Chemother. 2019;74(Suppl 1):i6-15.

73. Ianiro G, Tilg H, Gasbarrini A. Antibiotics as deep modulators of gut microbiota: between

76. Murphy EF, Cotter PD, Hogan A et al. Divergent metabolic outcomes arising from targeted manipulation of the gut microbiota in diet-induced obesity. Gut. 2013;62(2):220 LP - 226.

544 77. Hwang I, Park YJ, Kim Y-R et al. Alteration of gut microbiota by vancomycin and bacitracin improves insulin resistance via glucagon-like peptide 1 in diet-induced obesity. FASEB J. 2015;29(6):2397-411. 
547 78. Dao MC, Everard A, Aron-Wisnewsky J et al. Akkermansia muciniphila and improved 548 metabolic health during a dietary intervention in obesity: Relationship with gut microbiome 549 richness and ecology. Gut. 2016;65(3):426-36.

550 79. Bailey LC, Forrest CB, Zhang P et al. Association of Antibiotics in Infancy With Early 551 Childhood Obesity. JAMA Pediatr. 2014;168(11):1063-9.

552 80. Isanaka S, Langendorf C, Berthé $\mathrm{F}$ et al. Routine Amoxicillin for Uncomplicated Severe Acute Malnutrition in Children. N Engl J Med. 2016;374(5):444-53.

554 81. Eiseman B, Silen W, Bascom GS, Kauvar AJ. Fecal enema as an adjunct in the treatment of pseudomembranous enterocolitis. Surgery. 1958;44(5):854-9.

556 82. Vrieze A, Out C, Fuentes S et al. Impact of oral vancomycin on gut microbiota, bile acid metabolism, and insulin sensitivity. J Hepatol. 2014;60(4):824-31.

558 83. Chen K, Zheng X, Feng M, Li D, Zhang H. Gut Microbiota-Dependent Metabolite Trimethylamine N-Oxide Contributes to Cardiac Dysfunction in Western Diet-Induced Obese Mice. Front Physiol. 2017;8:139. 


\section{TABLES}

Table 1: Methods for gut microbiota analysis and their definitions

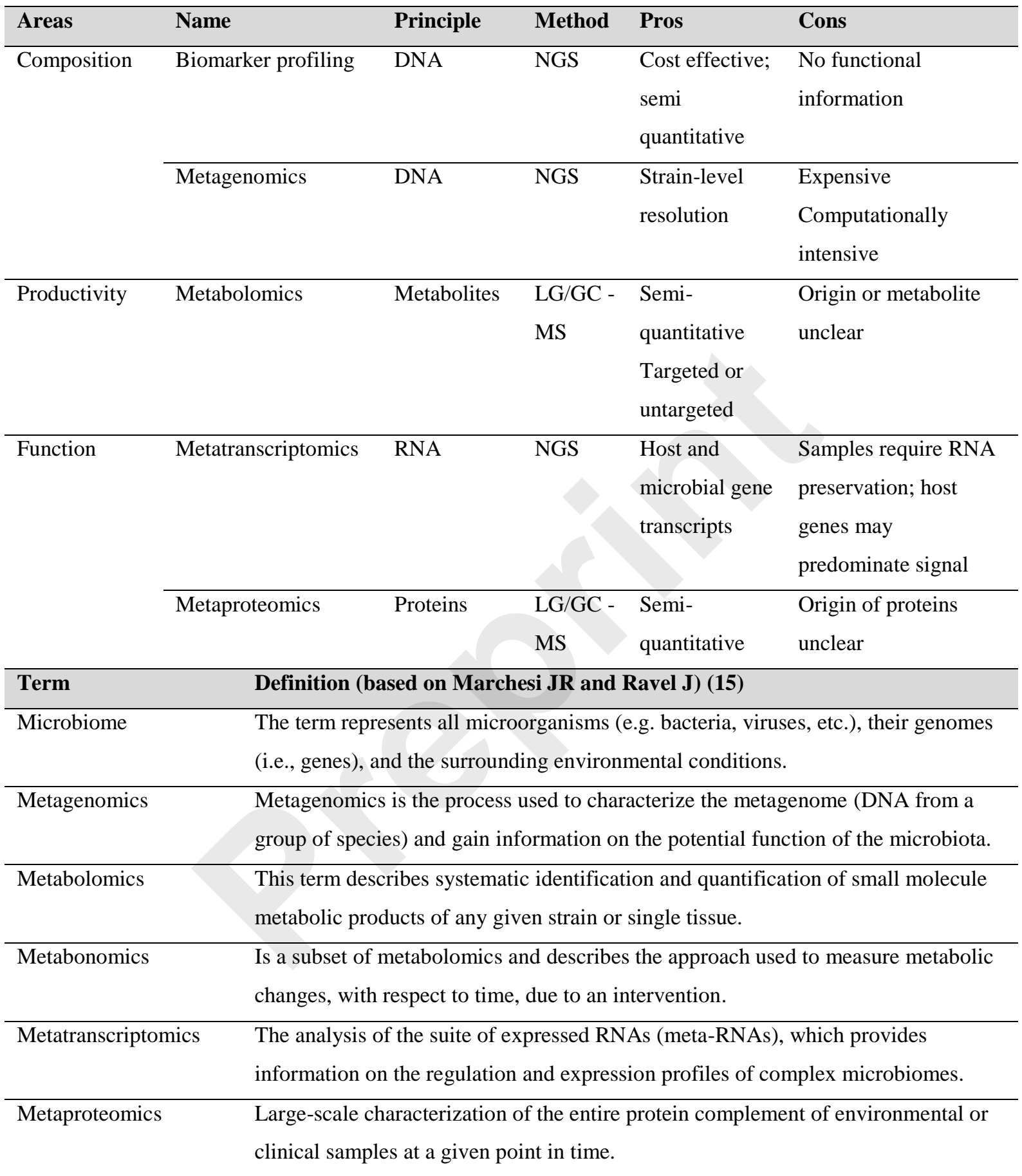

Legend: NGS - next-generation sequencing, LG/GC - liquid/gas chromatography, MS -

mass spectrometry. First part adapted from Durack J and Lynch SV (16). 


\section{FIGURE LEGENDS}

Figure 1: Microbial metabolites

Depiction of different microbial metabolites that trigger specific pathophysiological mechanisms in the development of cardiovascular diseases.

Figure 2: Gut hypothesis

The graphical depiction of the potential link between dysbiosis and heart failure.

Figure 3: Leaky gut hypothesis

The simplified graphical depiction of the potential link between dybiosis, the disruption of tight junction integrity and inflammatory response.

Figure 4: Effects of antibiotics on the microbiota composition

The depiction of the overall changes of specific antibiotic groups on the microbiota. Adapted from Bhalodi et al. (72) and Ianiro et al. (73). 


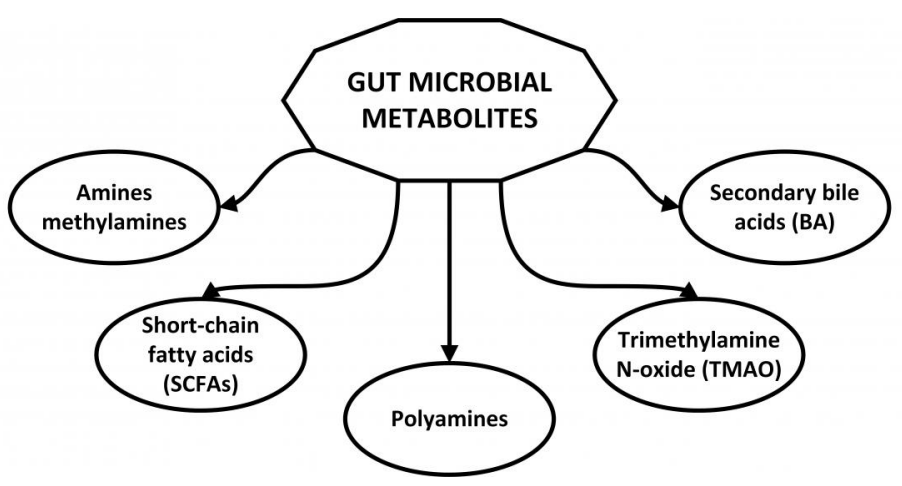

Figure 1: Microbial metabolites

Depiction of different microbial metabolites that trigger specific pathophysiological mechanisms in the development of cardiovascular diseases. 


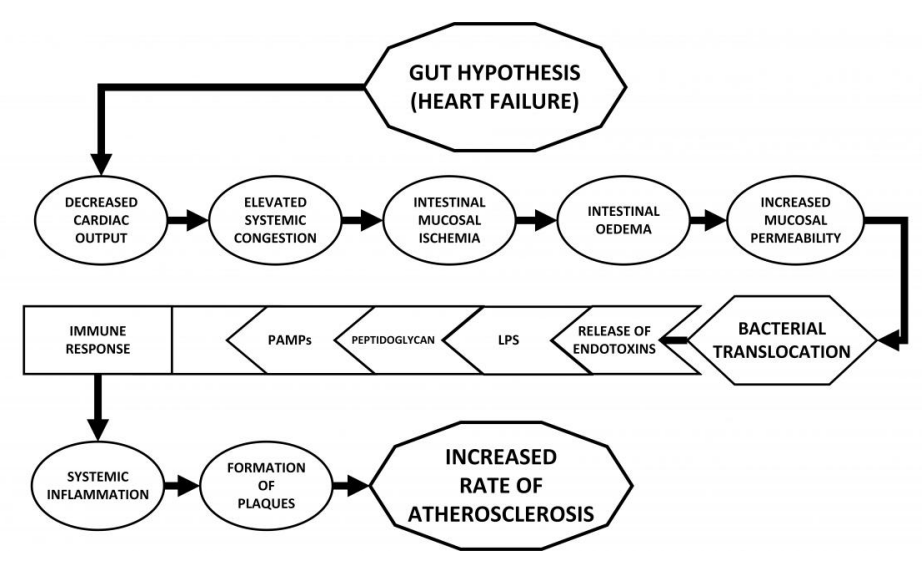

Figure 2: Gut hypothesis The graphical depiction of the potential link between dysbiosis and heart failure. 


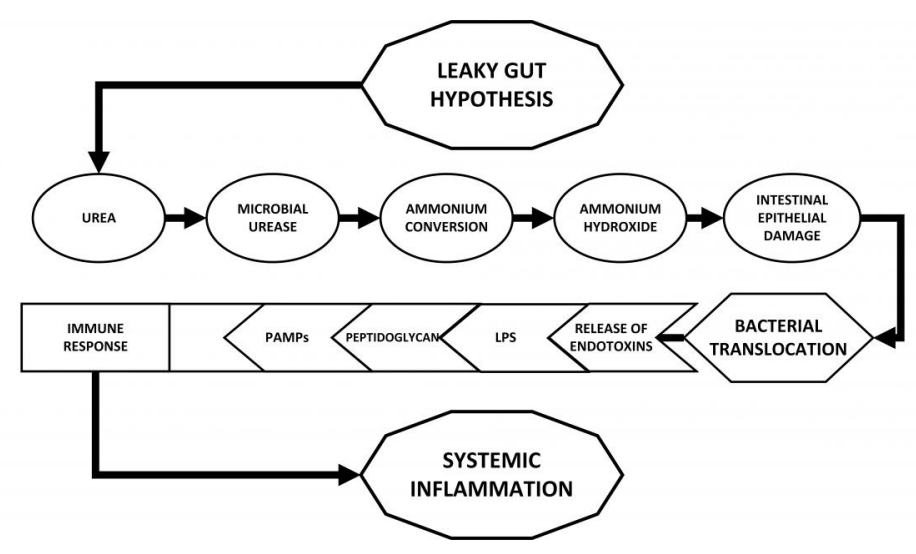

Figure 3: Leaky gut hypothesis The simplified graphical depiction of the potential link between dybiosis, the disruption of tight junction integrity and inflammatory response. 


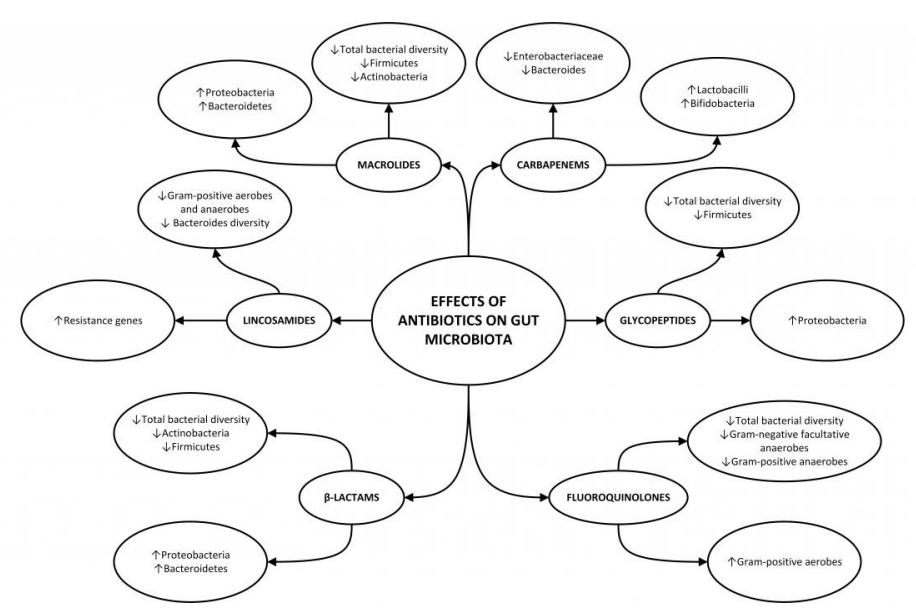

Figure 4: Effects of antibiotics on the microbiota composition The depiction of the overall changes of specific antibiotic groups on the microbiota. Adapted from Bhalodi et al. (72) and laniro et al. (73). 